\title{
Fluconazole prophylaxis for prevention of invasive candidiasis in infants
}

\author{
Jessica E. Ericson, MD ${ }^{1,2}$ and Daniel K. Benjamin Jr., MD, PhD, MPH ${ }^{1,2}$ \\ ${ }^{1}$ Duke Clinical Research Institute, Durham, NC \\ ${ }^{2}$ Department of Pediatrics, Duke University, Durham, NC
}

\begin{abstract}
Purpose of review-Invasive candidiasis is a serious infection in hospitalized infants that results in significant mortality and morbidity. Fluconazole is approved by the U.S. Food and Drug Administration for prophylaxis of invasive candidiasis in patients undergoing bone marrow transplantation but is not approved for use in infants. This review will describe the history of fluconazole use for prophylaxis in infants.
\end{abstract}

Recent findings-Limiting fluconazole prophylaxis to infants with risk factors, in addition to low birth weight and early gestational age, reduces the number of infants treated with fluconazole and the duration of fluconazole therapy for each infant.

Summary-Fluconazole prophylaxis appears to be safe for use in premature infants. Reduction in the incidence of invasive candidiasis is observed even when prophylaxis is limited to infants with multiple risk factors. Centers with a low incidence of invasive candidiasis may not benefit from fluconazole prophylaxis. Significant short- and long-term toxicity and increases in fluconazole-resistant organisms have not been observed with fluconazole use in the intensive care nursery.

\section{Keywords}

fungal infection; neonate; premature

\section{INTRODUCTION}

\begin{abstract}
Invasive candidiasis (IC) is associated with significant morbidity and mortality in premature infants. Among extremely low birth weight infants (ELBW, <1000 g birth weight) with IC, the mortality rate approaches $30 \%$, and up to $70 \%$ of survivors develop long-term neurodevelopmental impairments $\left[1,2^{* *}\right]$. Premature infants with IC are more likely to develop periventricular leukomalacia, retinopathy of prematurity, and chronic lung disease [3].
\end{abstract}

\footnotetext{
Correspondence to: Daniel K. Benjamin Jr., MD, PhD, MPH, Professor, Duke University, Pediatrics, Duke Clinical Research Institute, P.O. Box 17969, Durham, NC 27715, USA. Tel.: 919668 8295. danny.benjamin@duke.edu.

None.
} 
The incidence of IC varies greatly by center, but Candida species are considered to be the third most common cause of bloodstream infection in hospitalized very low birth weight (VLBW, <1500 g birth weight) infants [4]. Individual neonatal intensive care units (NICUs) have reported incidences in ELBW infants ranging from 4-30\% [5-8]. VLBW and ELBW infants are at increased risk, with infants $<750 \mathrm{~g}$ birth weight disproportionally at risk [1]; the risk of IC is 3 -fold higher for infants $<750 \mathrm{~g}$ compared to infants with birth weights of $750-1000 \mathrm{~g}$ [9]. The incidence of IC for infants with birth weights of 1001-1500 $\mathrm{g}$ is approximately $1 \%[10,11]$. Once birth weight is considered, site variation [12] and exposure to broad-spectrum antibiotics confer the greatest risk of IC [1]. Variation between NICU sites can be marked and is likely influenced by variation in antibiotic use, infection control practices, and central line care policies. Odds of IC can be as much as 6 times greater between 2 individual NICUs [12].

\section{PROPHYLAXIS: CAN IC BE PREVENTED?}

Due to the severe consequences of IC in premature infants, interventions aimed at primary prevention have been sought. Use of nystatin as antifungal prophylaxis in 33 infants with birth weights <1250 g was first published in 1988 [13]. The incidence of IC was lower in the group treated with nystatin (6\%) compared with placebo (32\%). Nystatin prophylaxis in VLBW infants was associated with a reduction in the incidence of IC from $14 \%$ to $2 \%$ when compared with no intervention [14].

Use of fluconazole for IC prophylaxis was first reported in VLBW infants in 2001 (Table 1). The first trial was not powered to demonstrate a decrease in IC, but a significant reduction in Candida colonization was noted [6]. A single-center randomized trial of ELBW infants demonstrated a significant reduction in both fungal colonization and IC using $3 \mathrm{mg} / \mathrm{kg}$ of intravenous fluconazole [5]. The dosing interval varied by postnatal age from 24 hours to 72 hours, and infants were treated until they were 6 weeks of age. This same center performed a small masked placebo-controlled trial a few years later using $3 \mathrm{mg} / \mathrm{kg}$ given twice weekly to 41 ELBW infants [22]. In addition to placebo, they compared these patients to 40 infants dosed according to postnatal age and found a similar improvement in the incidence of IC, with fewer doses of fluconazole required to achieve the same benefit of prophylaxis [22].

Italian investigators performed a multicenter, randomized, double-blind, placebo-controlled trial of fluconazole in 322 VLBW infants [7]. This trial demonstrated a significant reduction in both colonization and IC at doses of $3 \mathrm{mg} / \mathrm{kg}$ and $6 \mathrm{mg} / \mathrm{kg}$ compared with placebo. Doses were given every 72 hours for the first 2 weeks, then every 48 hours. VLBW infants were treated for 4 weeks and ELBW infants for 6 weeks. As with the previous smaller trials, fluconazole was not associated with decreased mortality.

Despite these successful trials, clinicians did not uniformly institute fluconazole prophylaxis in all premature infants [23]. Questions remained regarding immediate drug toxicity, future effects on infant neurodevelopment, and selection for fluconazole-resistant Candida species [24]. Additionally, no reduction in mortality with fluconazole prophylaxis had been observed. 


\section{TARGETED PROPHYLAXIS}

Several researchers sought to minimize the number of infants given fluconazole prophylaxis by identifying those who would benefit the most while avoiding unnecessary drug exposure in infants at lower risk. Bertini et al limited fluconazole prophylaxis to VLBW infants with central vascular access [15]. This strategy resulted in $62 \%$ fewer infants treated with fluconazole. Of 360 VLBW infants admitted during the study period, 136 (38\%) qualified for prophylaxis. IC occurred in 9 of $119(8 \%)$ historical infants given no fluconazole prophylaxis and 0 of $136(0 \%)$ once fluconazole prophylaxis was implemented.

Exposure to antibiotics was also used to select infants for antifungal prophylaxis. One study limited fluconazole prophylaxis to only VLBW infants receiving $>3$ days of antibiotic therapy [17]. This limited total fluconazole exposure to a median of 8 doses per infant compared with $>20$ doses per infant used in dosing regimens that treated all VLBW infants for 4-6 weeks $[5,7,16]$. The incidence of IC among all infants admitted to the NICU during the "fluconazole epoch" was compared with that of the "control epoch." Though fewer infants were given fluconazole than when a birth weight-based selection strategy was used, this approach decreased IC (1\%) compared with historical controls who did not receive fluconazole (6\%) [17]. McCrossan et al limited prophylaxis to VLBW infants with at least 1 of the following 3 criteria: treatment with a third-generation cephalosporin, $>10$ days of another broad-spectrum antibiotic, or evidence of fungal colonization if a central venous catheter was present. This cohort experienced a decrease in IC from 18\% (6/33) during a historical period to $0 \%(0 / 31)$ during the study period [18].

A large tertiary pediatric medical center in Israel also implemented a risk-based fluconazole prophylaxis protocol [21]. Risk factors were categorized as major or minor. One major criterion or 2 minor criteria triggered fluconazole prophylaxis. Major criteria included: birth weight $<1000 \mathrm{~g}$, corrected gestational age $<28$ weeks, and use of broad-spectrum antibiotics. Minor criteria included the presence of a central venous line, respiratory support, inotropic support, use of parenteral nutrition, use of a histamine-2 blocker or systemic steroids, confirmed necrotizing enterocolitis, or recent abdominal surgery. Using birth weight $<1000$ $\mathrm{g}$ and gestational age $<28$ weeks as major criteria resulted in the patient population receiving fluconazole in similar numbers to those of the original fluconazole prophylaxis trial [5] that treated all VLBW infants. However in this study, the infants were not obligated to complete a predetermined duration of prophylaxis. Rather, once they no longer met criteria through maturation or growth, the prophylaxis was stopped. One hundred and thirty infants received prophylaxis and were compared with 319 matched historical controls. This approach to prophylaxis reduced the incidence Candida bloodstream infections to $0.8 \%$ and reduced both the number of infants treated and the mean duration of therapy compared with birthweight prophylaxis strategies.

A recent trial used fluconazole prophylaxis in VLBW infants, $<6$ weeks postnatal age, receiving broad-spectrum antibiotics for $>2$ days plus 1 additional risk factor: presence of a central venous catheter, endotracheal intubation, necrotizing enterocolitis, or parenteral nutrition administration [20]. Fluconazole prophylaxis was withdrawn when the infant no longer met these criteria. This strategy resulted in approximately $30 \%$ of VLBW infants 
receiving fluconazole prophylaxis for a median duration of 7 days. Comparatively, fluconazole strategies targeting only birth weight $<1500 \mathrm{~g}$ would have treated $100 \%$ of the VLBW infants for 4-6 weeks. Fluconazole prophylaxis given according to this method of risk stratification reduced the incidence of IC in VLBW infants to $6 \%$ from $15 \%$ but failed to demonstrate a survival benefit.

\section{ONGOING CONCERNS}

All of the studies assessing targeted fluconazole prophylaxis used historical controls as the comparator group. As many factors affect the incidence of IC, it is impossible for these studies to fully determine the effect of fluconazole on the incidence of IC. Many centers have reported dramatic decreases in overall bloodstream infection incidence through the use of bundles for central line insertion and maintenance, dedicated line-care personnel, and increased use of breast milk for enteral nutrition [25-28]. Similarly, the incidence of Candida central line-associated bloodstream infections has decreased over the course of 10 years even in birth weight cohorts that are not considered for fluconazole prophylaxis (e.g., $>1000$ or $>1500 \mathrm{~g}$ birth weight) [29*].

Additionally, many studies of fluconazole prophylaxis have come from centers with high baseline incidences of IC. The national incidence of IC in ELBW infants is 7\% [4,9]. Several study populations had a pre-fluconazole prophylaxis IC incidence that was much higher than that seen nationally $(15-20 \%)[5,7,18,20]$. While a reduction in IC incidence with fluconazole prophylaxis is approximately $80 \%$, it is unclear if the benefits of routine prophylaxis will be clinically meaningful in low-incidence settings.

The potential for selection of resistant Candida isolates has also been a concern surrounding implementation of widespread antifungal prophylaxis, although this has not yet been reported in the intensive care nursery. A comparison of the resistance patterns of isolates causing IC during the 2 years before and the 4 years after the initiation of a fluconazole prophylaxis protocol was reported at a single center [30]. The number of infections caused by fluconazole-resistant Candida species after initiation of fluconazole prophylaxis was similar to that seen in the 2 years prior to implementation. Another study also found no increase in inherently fluconazole-resistant Candida species when the incidence before routine fluconazole prophylaxis for VLBW infants was compared with that after prophylaxis was initiated [31*]. These studies are reassuring but are limited by their relatively short duration and small samples sizes. The effect of a prolonged fluconazole prophylaxis strategy, which may be used indefinitely into the future in thousands of infants, remains to be seen. Intermittent prophylaxis has been proposed [32], but this strategy may introduce resistance as the serum fluconazole concentration intermittently falls below the minimum inhibitory concentration.

While the outcomes following IC in infants are poor $[1,2 * *]$, the long-term consequences of exposure to fluconazole, in many cases for several weeks, is a concern for those considering implementation of routine prophylaxis protocols. ELBW infants involved in a randomized, placebo-controlled fluconazole prophylaxis trial [5] were recently assessed for neurodevelopmental, behavioral, and quality-of-life outcomes at 8-10 years of age [33**]. 
Twenty children who had received fluconazole as infants did not differ in any of the tested domains from 17 who were given placebo. While reassuring, this was a small study and may be biased by differential loss to follow-up giving the long time frame.

\section{CONCLUSION}

Evidence accumulated over the last 13 years suggests that fluconazole prophylaxis for the prevention of IC is effective and safe in premature infants. Risk-based selection strategies are likely the best way to balance the real risk that IC poses to premature infants with the risks that are inherent to any drug therapy. Risk stratification should be primarily focused on the NICU where the infant is hospitalized [34]. Prophylaxis is unlikely to be of substantial benefit at centers where the rate of IC $<5 \%$ at baseline. European guidelines have suggested that a $2 \%$ incidence be the threshold for implementing a fluconazole prophylaxis program [35**]. A threshold of $2-5 \%$ for implementing prophylaxis is a reasonable approach that balances the risks associated with additional therapeutics and the benefits of preventing IC. Continued surveillance for changes in antifungal susceptibility patterns should be performed.

\section{Acknowledgments}

Dr. Benjamin receives support from the United States government for his work in pediatric and neonatal clinical pharmacology (1R01HD057956-05, 1K24HD058735-05, and NICHD contract HHSN275201000003I) and the nonprofit organization Thrasher Research Fund for his work in neonatal candidiasis (www.thrasherresearch.org); he also receives research support from industry for neonatal and pediatric drug development (www.dcri.duke.edu/ research/coi.jsp). Dr. Ericson receives support from the NIH through an institutional training grant (5T32HD060558-03, PI Cunningham).

\section{ABBREVIATIONS}
ELBW Extremely low birth weight
IC Invasive candidiasis
NICU Neonatal intensive care unit
VLBW Very low birth weight

\section{REFERENCES AND RECOMMENDED READING}

Papers of particular interest, published within the annual review period, have been highlighted as *special interest and **of outstanding interest.

1. Benjamin DK Jr, Stoll BJ, Fanaroff AA, et al. Neonatal candidiasis among extremely low birth weight infants: risk factors, mortality rates, and neurodevelopmental outcomes at 18 to 22 months. Pediatrics. 2006; 117:84-92. [PubMed: 16396864]

2**. Adams-Chapman I, Bann CM, Das A, et al. Neurodevelopmental outcome of extremely low birth weight infants with Candida infection. J Pediatr. 2013; 163:961-967. This thorough study compared performance on 18-month Bayley scales for ELBW infants with Candida sepsis to that of ELBW infants without sepsis. The odds of neurodevelopmental impairment for those with Candida sepsis was nearly twice that of infants without sepsis. [PubMed: 23726546]

3. Friedman S, Richardson SE, Jacobs SE, et al. Systemic Candida infection in extremely low birth weight infants: short term morbidity and long term neurodevelopmental outcome. Pediatr Infect Dis J. 2000; 19:499-504. [PubMed: 10877162] 
4. Stoll BJ, Hansen N, Fanaroff AA, et al. Late-onset sepsis in very low birth weight neonates: the experience of the NICHD Neonatal Research Network. Pediatrics. 2002; 110(2 Pt 1):285-291. [PubMed: 12165580]

5. Kaufman D, Boyle R, Hazen KC, et al. Fluconazole prophylaxis against fungal colonization and infection in preterm infants. N Engl J Med. 2001; 345:1660-1666. [PubMed: 11759644]

6. Kicklighter SD, Springer SC, Cox T, et al. Fluconazole for prophylaxis against candidal rectal colonization in the very low birth weight infant. Pediatrics. 2001; 107:293-298. [PubMed: 11158461]

7. Manzoni P, Stolfi I, Pugni L, et al. A multicenter, randomized trial of prophylactic fluconazole in preterm neonates. N Engl J Med. 2007; 356:2483-2495. [PubMed: 17568029]

8. Violaris K, Carbone T, Bateman D, et al. Comparison of fluconazole and nystatin oral suspensions for prophylaxis of systemic fungal infection in very low birthweight infants. Am J Perinatol. 2010; 27:73-78. [PubMed: 19504425]

9. Benjamin DK Jr, Stoll BJ, Gantz MG, et al. Neonatal candidiasis: epidemiology, risk factors, and clinical judgment. Pediatrics. 2010; 126:e865-873. [PubMed: 20876174]

10. Fridkin SK, Kaufman D, Edwards JR, et al. Changing incidence of Candida bloodstream infections among NICU patients in the United States: 1995-2004. Pediatrics. 2006; 117:1680-1687. [PubMed: 16651324]

11. Manzoni P, Arisio R, Mostert M, et al. Prophylactic fluconazole is effective in preventing fungal colonization and fungal systemic infections in preterm neonates: a single-center, 6-year, retrospective cohort study. Pediatrics. 2006; 117:e22-32. [PubMed: 16326690]

12. Cotten CM, McDonald S, Stoll B, et al. The association of third-generation cephalosporin use and invasive candidiasis in extremely low birth-weight infants. Pediatrics. 2006; 118:717-722. [PubMed: 16882828]

13. Sims ME, Yoo Y, You H, et al. Prophylactic oral nystatin and fungal infections in very-lowbirthweight infants. Am J Perinatol. 1988; 5:33-36. [PubMed: 3276336]

14. Ozturk MA, Gunes T, Koklu E, et al. Oral nystatin prophylaxis to prevent invasive candidiasis in the neonatal intensive care unit. Mycoses. 2006; 49:484-492. [PubMed: 17022766]

15. Bertini G, Perugi S, Dani C, et al. Fluconazole prophylaxis prevents invasive fungal infection in high-risk, very low birth weight infants. J Pediatr. 2005; 147:162-165. [PubMed: 16126042]

16. Healy CM, Baker CJ, Zaccaria E, et al. Impact of fluconazole prophylaxis on incidence and outcome of invasive candidiasis in a neonatal intensive care unit. J Pediatr. 2005; 147:166-171. [PubMed: 16126043]

17. Uko S, Soghier LM, Vega M, et al. Targeted short-term fluconazole prophylaxis among very low birth weight and extremely low birth weight infants. Pediatrics. 2006; 117:1243-1252. [PubMed: 16585321]

18. McCrossan BA, McHenry E, O’Neill F, et al. Selective fluconazole prophylaxis in high-risk babies to reduce invasive fungal infection. Arch Dis Child Fetal Neonatal Ed. 2007; 92:F454-458. [PubMed: 17460023]

19. Aziz M, Patel AL, Losavio J, et al. Efficacy of fluconazole prophylaxis for prevention of invasive fungal infection in extremely low birth weight infants. Pediatr Infect Dis J. 2010; 29:352-356. [PubMed: 19934791]

20. Martin A, Pappas A, Lulic-Botica M, et al. Impact of 'targeted' fluconazole prophylaxis for preterm neonates: efficacy of a highly selective approach? J Perinatol. 2012; 32:21-26. [PubMed: 21760586]

21. Rolnitsky A, Levy I, Sirota L, et al. Targeted fluconazole prophylaxis for high-risk very low birth weight infants. Eur J Pediatr. 2012; 171:1481-1487. [PubMed: 22628137]

22. Kaufman D, Boyle R, Hazen KC, et al. Twice weekly fluconazole prophylaxis for prevention of invasive Candida infection in high-risk infants of <1000 grams birth weight. J Pediatr. 2005; 147:172-179. [PubMed: 16126045]

23. Burwell LA, Kaufman D, Blakely J, et al. Antifungal prophylaxis to prevent neonatal candidiasis: a survey of perinatal physician practices. Pediatrics. 2006; 118:e1019-1026. [PubMed: 16982807] 
24. Hachem R, Hanna H, Kontoyiannis D, et al. The changing epidemiology of invasive candidiasis: Candida glabrata and Candida krusei as the leading causes of candidemia in hematologic malignancy. Cancer. 2008; 112:2493-2499. [PubMed: 18412153]

25. Schulman J, Stricof R, Stevens TP, et al. Statewide NICU central-line-associated bloodstream infection rates decline after bundles and checklists. Pediatrics. 2011; 127:436-444. [PubMed: 21339265]

26. Wirtschafter DD, Powers RJ, Pettit JS, et al. Nosocomial infection reduction in VLBW infants with a statewide quality-improvement model. Pediatrics. 2011; 127:419-426. [PubMed: 21339273]

27. Aly H, Herson V, Duncan A, et al. Is bloodstream infection preventable among premature infants? A tale of two cities. Pediatrics. 2005; 115:1513-1518. [PubMed: 15930211]

28. Hylander MA, Strobino DM, Dhanireddy R. Human milk feedings and infection among very low birth weight infants. Pediatrics. 1998; 102:E38. [PubMed: 9724686]

29*. Chitnis AS, Magill SS, Edwards JR, et al. Trends in Candida central line-associated bloodstream infections among NICUs, 1999-2009. Pediatrics. 2012; 130:e46-52. Catheter-related candidemia has decreased among all birth weight cohorts over the last 10 years. [PubMed: 22711720]

30. Healy CM, Campbell JR, Zaccaria E, et al. Fluconazole prophylaxis in extremely low birth weight neonates reduces invasive candidiasis mortality rates without emergence of fluconazole-resistant Candida species. Pediatrics. 2008; 121:703-710. [PubMed: 18381534]

31*. Manzoni P, Leonessa M, Galletto P, et al. Routine use of fluconazole prophylaxis in a neonatal intensive care unit does not select natively fluconazole-resistant Candida subspecies. Pediatr Infect Dis J. 2008; 27:731-737. This single-center study suggests that increases in fluconazoleresistant Candida species are unlikely to occur when fluconazole is routinely given for prophylaxis. [PubMed: 18600191]

32. Revankar SG, Kirkpatrick WR, McAtee RK, et al. A randomized trial of continuous or intermittent therapy with fluconazole for oropharyngeal candidiasis in HIV-infected patients: clinical outcomes and development of fluconazole resistance. Am J Med. 1998; 105:7-11. [PubMed: 9688014]

33**. Kaufman DA, Cuff AL, Wamstad JB, et al. Fluconazole prophylaxis in extremely low birth weight infants and neurodevelopmental outcomes and quality of life at 8 to 10 years of age. $\mathrm{J}$ Pediatr. 2011; 158:759-765. This long-term follow-up study of VLBW infants given fluconazole for IC prophylaxis found that 8-10-year outcomes were similar between those given placebo and those given fluconazole. [PubMed: 21168853]

34. Pappas PG, Kauffman CA, Andes D, et al. Clinical practice guidelines for the management of candidiasis: 2009 update by the Infectious Diseases Society of America. Clin Infect Dis. 2009; 48:503-535. [PubMed: 19191635]

35**. Hope WW, Castagnola E, Groll AH, et al. European Society of Clinical Microbiology and Infectious Diseases guideline for the diagnosis and management of Candida diseases 2012: prevention and management of invasive infections in neonates and children caused by Candida spp. Clin Microbiol Infect. 2012; 18 (Suppl 7):38-52. Guidelines for the management of Candida infections in children suggest that fluconazole prophylaxis be implemented in NICUs that have an incidence of IC greater than 2\%. x. [PubMed: 23137136] 


\section{KEY POINTS}

- Fluconazole prophylaxis appears to be safe and reduces the incidence of IC.

- Fluconazole prophylaxis should be limited to high-risk infants admitted to highincidence NICUs.

- A dosing regimen of $6 \mathrm{mg} / \mathrm{kg}$ twice weekly is adequate when fluconazole is used for prophylaxis. 


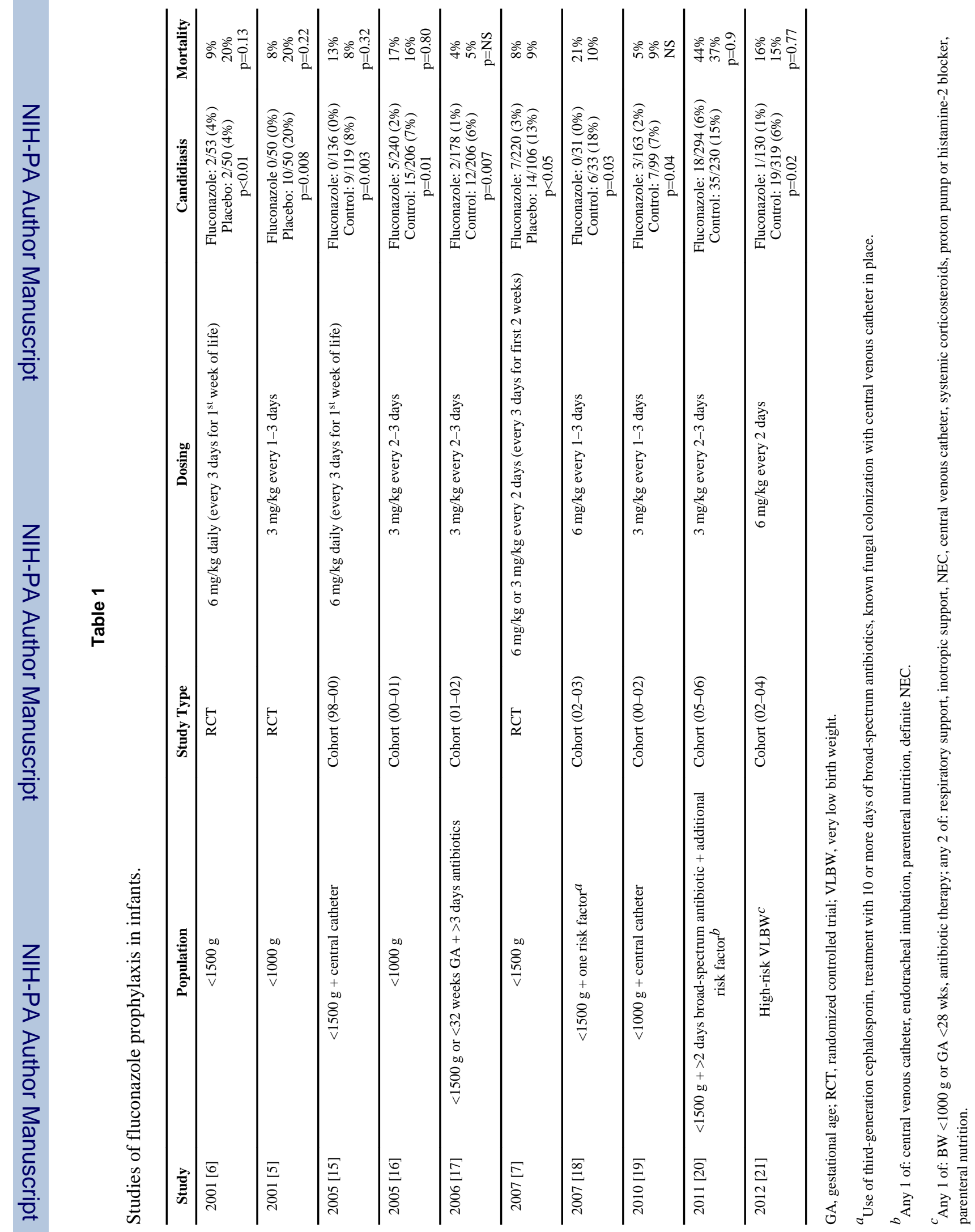

